\title{
Writing high-quality statistical software for Apple II microcomputers
}

\author{
DARRELL L. BUTLER \\ Ball State University, Muncie, Indiana \\ and \\ DOUGLAS B. EAMON \\ University of Wisconsin-Whitewater, Whitewater, Wisconsin
}

\begin{abstract}
The purpose of this paper is to describe some principles and techniques that programmers should consider when trying to improve or create a statistical package. Four computational issues are discussed: accuracy, speed, computing probabilities, and error trapping. A single-array data structure is proposed for use within the program. Several other input-output issues are covered, including menus, command languages, questions, data input and editing, CRT and printer formatting, and graphics.
\end{abstract}

Recent reviews of statistical packages for microcomputers (e.g., Butler, 1986; Butler \& Eamon, 1985; Butler \& Jones, 1987; Carpenter, Deloria, \& Morganstein, 1984; Eamon, 1983; Francis, 1983; Lehman, 1986) indicate that there are some good packages available on the market but few, if any, outstanding low-cost packages. Thus some researchers may wish to adapt purchased software or create their own. Wolach's (1983) book on writing one's own statistical software is limited to analysis of variance (ANOVA), and some of the programs contain bugs.

Although ANOVA is very useful, other routines are often needed. The purpose of this paper is to provide some principles that programmers should consider when trying to adapt or create statistical packages. The Apple II series computer is used as a framework because it is still the most widely used computer. However, most, if not all, of the principles discussed here are useful to programmers working on other computer systems.

The principles are divided into the following categories: computations, data structures, and input/output. Each of these is discussed below.

\section{COMPUTATION}

If statistical software is to be useful, it must be reasonably accurate and fast. An additional advantage is gained if the package contains computation of probabilities. One other issue considered below is the importance of error trapping in computations.

\section{Accuracy}

Simple sums, rank orders, and counting can be done quite accurately on the Apple II, at least with the kinds

Requests for reprints should be sent to the first author at the Department of Psychological Sciences, Ball State University, Muncie, IN 47306. of dependent variables typically used by psychologists. Accuracy problems occur when numbers are squared or higher powers are calculated. This problem is most acute with sums of squares, a common calculation used in computational formulas of variances, standard deviations, $t$ tests, and ANOVAs. These computational formulas are inappropriate for computer programs. Accuracy is better if definition formulas or updating formulas are used.

The definition formula for the variance is:

$$
\Sigma(X-M)^{2} / N \text {, }
$$

where $X s$ are the scores, $M$ is the mean of all scores, and $N$ is the number of scores. To calculate this formula in a program requires one pass through the data to obtain the mean and a second pass to compute the variance. Although the two passes can take time, the result is very accurate.

One kind of calculating formula, an updating formula, is useful for computer calculation. The goal is accuracy with a single pass through the data. An example for sum of squared deviations from the mean is

$$
V+(X-M)^{2} \frac{N}{N+1},
$$

where $V$ is the old sum of squared deviations (before the new score $X$ is included), $M$ is the old mean, and $N$ is the old number of scores. To use this formula, set $V=0$, $M=0$, and $N=0$ and then use this updating formula in a loop. After all data has been included, divide by $N$ (or $N-1$ ) to obtain the variance. An example using this equation is given in Table 1 along with an equation that updates a variance when a score is removed from a set of data.

Note that the definition and updating formulas can be generalized for use in ANOVA and regression. In ANOVA, each term in the ANOVA summary table represents a partition of the variance (see Hays, 1981, 
Table 1

Updating Variance and Mean by Adding or Deleting a Score

Adding Scores
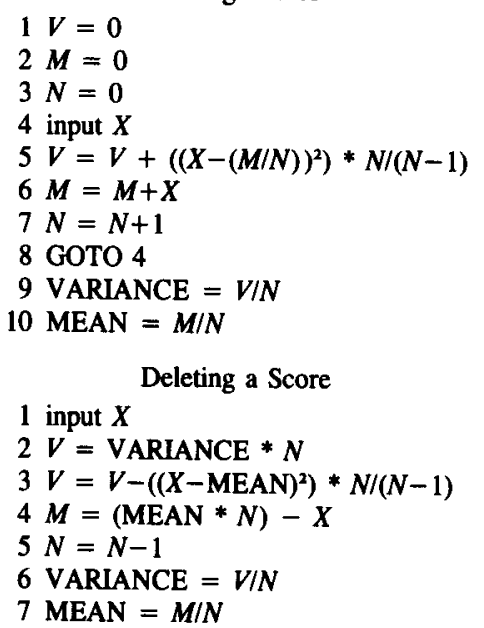

Note $-X=$ score; $M=$ the sum of all scores; $N=$ the number of scores; $V=$ the old sum of squared deviations.

p. 336). Using the definitional formula, the mean square between groups is

$$
\Sigma\left[N \mathrm{j}(\mathrm{Xj}-M)^{2}\right] / J
$$

where $N \mathrm{j}$ represents the number of scores in group $\mathrm{j}, X \mathrm{j}$ is the mean of group $\mathrm{j}, J$ is the number of groups, and $M$ is the mean of all scores combined. The formula above produces greater accuracy on a computer than does the more common calculation formula:

$$
\frac{\Sigma(\Sigma X)^{2}}{N \mathrm{j}}-\frac{(\Sigma \Sigma X)^{2}}{N}
$$

\section{Speed}

Speed is not always a high priority, but typically it is not irrelevant. When many programmers think of increasing speed, they think of using machine code. This is certainly an option, but there are also others: doing computations during input, optimizing the code, and compiling.

Some computations can be done quickly during input (e.g., a sum of a group of scores, an updated mean, or an updated variance can be obtained). Since typing is typically slow, these calculations are transparent to users. As shown in Table 1, updated means and variances can also be obtained during editing of data. Remove deleted or changed scores and add contributions of added or altered scores.

Program code can be optimized following good programming principles. For example, variables should be used instead of constants in Apple BASIC, especially in loops. Eamon and Butler (1987) provide a relatively complete list of optimizing principles, along with other suggestions for increasing speed of programs.

Speed can also be increased in statistics programs by selecting good computation algorithms. For example, Zar (1985) has published a fast sorting algorithm that can be useful in programs requiring ordered data. The journals of the American Statistical Association and Behavior Research Methods, Instruments, \& Computers regularly publish potentially useful algorithms and short programs.

\section{Probabilities}

There is no reason why the computer cannot provide reasonably accurate probability values for statistics. Many common statistics can be related to the normal distribution (z). B. J. R. Bailey (1980) provides several relatively accurate transformations of Student's $t$ to $z$. For example,

$$
z= \pm\{(8 v+1)(8 v+9)\}\left[(v+19 / 12) \log \left\{1+t^{2}(v+1 / 12)\right\}\right]^{1 / 2},
$$

where $v$ is the number of degrees of freedom. This function is designed to be especially accurate for $t$ values with probabilities around .05. Johnson and Kotz (1970) provide a transformation for $F$ to $z$ :

$$
z=\left[(1-2 / 9 d) F^{1 / 3}-(1-2 / 9 n)\right]\left[(2 / 9 d) F^{2 / 3}+(2 / 9 n)\right]^{-1 / 2},
$$

where $n$ is the degrees of freedom in the numerator of the $F$, and $d$ is the degrees of freedom in the denominator. Other distributions can be related to $F$ or $t$. For example,

$$
\frac{\chi^{2}}{d f}=F(d f, \infty) \text { and } t^{2}=\frac{r^{2}(N-2)}{\left(1-r^{2}\right)} .
$$

Thus it is possible to relate many common statistics to the $z$ distribution.

It is then possible to use an algorithm to approximate the probability of a $z$. Several statisticians (e.g., Lin, 1982) use the following approach:

$p=.5 /(1+z(.196854+z(.115194+z(.00344+.019527 z))))^{4}$.

Due to rounding error and limitations of these formulas, it may be necessary to add a "correction" in order to maximize accuracy around $p=.05$. For example, for some statistics we subtract .015 from $z$ when degrees of freedom are small because we have found it improves the approximation.

\section{Error Handling}

Errors that could cause program interruption must be trapped. One of the most common errors is dividing by zero because there is no data or no variance. Use "ONERR GOTO" statements as well as "IF"' statements to check for specific conditions. Appropriate messages should be printed, and the user should be given the option to reenter the program at a convenient spot.

\section{DATA STRUCTURES}

An advantage of microcomputer software over hand calculators is that multiple statistical procedures can be run on the same data, without having to reenter the data. A good data structure must not only permit flexibility, but also must be capable of handling a reasonable number of scores. Some programmers arbitrarily select a maximum number of scores for each group in the data structure (e.g., 
Eckblad, 1984). Others use multiple-dimensioned arrays (number of groups, number per group), thereby producing a trade-off between number of groups and number of scores per group (e.g., ANOVA routines in Statistical Programs for the Apple II by Steinmetz, 1981). A better option is to use a single array subdivided by pointers. The pointers indicate the array position of the beginning and end of each group of scores within the array. If a single array is used, the only limitation is the total number of data, not the largest number of scores in a group or equality of groups. For example, an array can have the following kind of organization:

$$
\begin{aligned}
& \text { number of groups }(N), \\
& \text { size of group } 1, \\
& \text { size of group } 2, \\
& \ldots \text {, } \\
& \text { size of group } N, \\
& \text { data .... }
\end{aligned}
$$

This data structure or similar ones that also contain group means and variances can be saved to a disk. This structure provides high compatibility among routines. All statistical procedures requiring two groups can use any array containing two or more groups. Thus the same data can be analyzed using a $t$ test and a Mann-Whitney $U$ without reentering data. Special checks on data can be added to routines to limit this compatibility. For example, chi-square tests use frequency data organized somewhat differently from that of most other procedures, and some procedures (e.g., dependent-groups $t$ test) require the same number of scores in each group. "IF" statements for specific characteristics of the data structure can be used to implement these limits.

Both sequential and random access disk files can be used. There are several advantages to sequential files: programs are simpler, a part of a file can be saved before the length of the entire file is known, and sequential files use disk space relatively efficiently. However, if very large data sets containing many variables are to be stored in a disk file, then random access files may be preferable.

Since so many different data structures are used by marketed packages, a useful procedure is one that transforms one data structure to another. Common data structures are DIF (used by many spreadsheet programs) and dataonly ASCII file (used by many statistics packages).

\section{INPUT/OUTPUT}

There are several issues concerning input/output: alphabetic interaction (menus, commands, and answers to questions), data input and editing, format of CRT and printer, and graphics.

\footnotetext{
Alphabetic Interaction

Menus are a highly desirable way to get some kinds of input from users (e.g., selection of a statistical procedure or selection from a set of options at the end of a procedure). They are user friendly when they are short,
}

written simply, and clear. They should not contain too many options, but they should contain options to back up in the program (i.e., return to an earlier point) or to escape from the program. The terminology should be carefully selected (use of pretests should be considered). The CRT should not be too cluttered and should be printed from top to bottom in a logical order. This will result in an easy-to-use program, both for those using CRTs and for those using speech synthesizers. Menu options should be numbered (or labeled some other way) so that options can be chosen by selecting a number or by using arrow keys. To select options, users should always select, then press return, since this will be the case in data input and consistency is desirable. You may also want to keep in mind compatibility with alternative interfaces, such as the mouse and speech recognizers, which are used by the visually and muscularly handicapped. Statistics software should be easy to make compatible with these alternative interfaces. Programmers should carefully trap input errors. Also, dynamic string arrays should be used as little as possible.

An alternative to menus is command languages. A drawback to command languages is that users must learn the commands. However, if commands are well chosen (as in MINITAB, a mainframe computer statistics package), this potential drawback is avoided. From a programmer's standpoint, command languages are more difficult to implement than are menus. Parsing of subject responses takes many lines of code. However, a well-conceived command language can provide an efficient, effective user-software interface.

Questions can be used to handle simple choices. Questions should be short and clear and should indicate possible responses (e.g., “Printer output (Y or N)?"). Responses should be single keypresses followed by a press on the return key, because such a system is consistent with menu selection and data input. Programmers should trap errors carefully.

\section{Numerical Input and Editing}

The CRT should indicate the number of the data item being input and the group number of the data if it is partitioned into groups. Non-numerical inputs should be trapped. Several techniques are useful. A machine language input routine can be used; some are marketed (see Eamon \& Butler, 1987). In BASIC, the GET command can be used to obtain input. Then inappropriate keypresses can be thrown away and feedback (e.g., bell) given to the user. Appropriate keypresses can be echoed to the CRT. An alternative in BASIC is to use an ONERR GOTO statement for error handling and then INPUT data in a string. The string can be converted to a number. All of these techniques work as long as error checking is thorough.

One advantage of working with software on a microcomputer over working with an inexpensive hand calculator is the ability to edit data. Many characteristics of good word processors and editors should be considered. 
On-screen editing (i.e., changing values with immediate feedback on the CRT) is highly desirable. It is easiest for users to have a small amount of data (numbered) on the display at one time. The user selects items by number or by moving a cursor to the correct position, and then makes the corrections. Insertions and deletions are more difficult to program than changing a value. Remember to keep the procedure simple from the user's point of view.

\section{CRT Format}

Basic rules for formatting information on CRTs have been discussed in detail (e.g., R. W. Bailey, 1982). Many of these rules can be included easily in a statistical program. Some specific rules for statistics should be considered.

A desirable feature is to have a primary summary of the statistics on one or two pages and optional pages containing more preliminary calculations or advanced calculations. For example, the primary page can list the correlation coefficient with some other information; a secondary page can list variances, sums of squares, etc.; and another secondary page can be used for significance tests of slope, covariances, etc. The secondary pages contain information only a few users would probably want.

Screen and printer output can be quite different because some Apple IIs have only 40-column CRT capability, but a greater number of columns can be printed. Programmers should not assume printers have more than 80 columns. Separate routines for each type of output should be considered. Computer hangs that result from calling the printer should be trapped so that data is not lost. This can be done in several ways, one of which is to print a message on the CRT telling users what to do if the computer hangs (e.g., "If computer quits working, press control reset, type GOTO 193, and press return'). Select a reentry position that will not erase data. An alternative is to simply print "If computer quits working, press control reset" and then trap the reset key and branch the user to the appropriate location selected. Branching is not very easy to do however.

\section{Graphics}

Graphics may not be a requirement of a statistics package, but they are a valuable adjunct and an advantage of microcomputer software over hand calculators. The ability to produce and save a scatter plot, a bar graph, and line chart is desirable. Few inexpensive programs presently include graphics, and the graphics in some packages are poor. Kosslyn (1985) recently reviewed five books on graphing data. This review and the books discussed in the review are worth the time required to find them. Implementing the ideas expressed in these sources and making graphs without abundant user-computer interaction could make a statistical package much more useful.

\section{REFERENCES}

BAILEY, B. J. R. (1980). Accurate normalizing transformations of a Student's $t$ variate. Applied Statistics, 29, 304-306.

BAILEY, R. W. (1982). Human performance engineering: A guide for system designers. Englewood Cliffs, NJ: Prentice-Hall.

BUTLER, D. L. (1986). Elementary statistical packages for microcomputers. Contemporary Psychology, 31, 485-487.

Butler, D. L., EAMON, D. B. (1985). An evaluation of statistical software for research and instruction. Behavior Research Methods, Instruments, \& Computers, 17, 352-358.

ButLer, D. L., \& JoNES, S. K. (1987). A comparison of inexpensive statistical packages for Apple II microcomputers. Behavior Research Methods, Instruments, \& Computers, 19, 99-103.

Carpenter, J., Deloria, D., Morganstein, D. (1984). Statistical software for microcomputers. BYTE, 9(4), 234-364.

EAMON, D. B. (1983). Microcomputer programs for instruction in psychology. Collegiate Microcomputer, 1, 11-18.

EAmon, D. B., ButLeR, D. L. (1987). Writing programs on the Apple for student psychology laboratories: Routines, subroutines, and sources of information. Behavior Research Methods, Instruments, \& Computers, 19, 88-98.

ECKBLAD, J. (1984). Keystat [Computer program]. Decorah, IA: Oakleaf Systems.

Francis, I. (1983). A survey of statistical software. Computational Statistics \& Data Analysis, 1, 17-27.

Hays, W. L. (1981). Statistics (3rd ed.). New York: Holt, Rinehart, \& Winston.

Johnson, N. L., \&otz, S. (1970). Continuous univariate distributions2. Boston, MA: Houghton-Mifflin.

KossLYN, S. M. (1985). Graphics and human information processing: A review of time books. Journal of the American Statistical Association, 80, 499-512.

Lehman, R. S. (1986). Macintosh statistical programs. Behavior Research Methods, Instruments, \& Computers, 18, 177-187.

LiN, S. P. (1982). Automatic model selection in contingency tables. Applied Statistics, 31, 317-326.

Steinmetz, J. (1981). Statistical programs for the Apple II [Computer program]. Athens, $\mathrm{OH}$ : Author.

WOLACH, A. H. (1983). BASIC analysis of variance programs for microcomputers. Monterey, CA: Brooks/Cole.

ZAR, J. H. (1985). Ranking data with BASIC. Behavior Research Methods, Instruments, \& Computers, 17, 142. 